\title{
On the Effects of Node Density and Duty Cycle on Energy Efficiency in Underwater Networks
}

\author{
Francesco Zorzi ${ }^{\dagger}$, Milica Stojanovic ${ }^{\ddagger}$ and Michele Zorzi ${ }^{\dagger}$ \\ $\dagger$ Dipartimento di Ingegneria dell'Informazione, Università degli Studi di Padova \\ Email: \{zorzifra,zorzi\}@ dei.unipd.it \\ $\ddagger$ Department of Electrical and Computer Engineering, Northeastern University, Boston. \\ Email: millitsa@ece.neu.edu
}

\begin{abstract}
Energy-efficiency in underwater networks is a key issue that affects all aspects of network design, from hardware to protocols and applications. In this paper we analyze the impact of node density on the energy consumption in transmission, reception and idle-listening, in a network where nodes follow a duty cycle scheme. We consider the energy performance of the network for different scenarios, where a different number of nodes and different values of the duty cycle are taken into account. We simulate different power settings, showing that there exists an effective network density for which the energy consumption is minimized.
\end{abstract}

\section{INTRODUCTION}

With emerging applications in areas such as environmental monitoring and underwater tracking, acoustic networks have raised interest in the scientific community. The variety of possible scenarios, including both static and mobile networks, with low or high traffic, call for the development of flexible networking solutions. Because of the substantial difference between wireless radio and underwater networks, recent research has addressed various aspects of communications and networking, from the physical layer to the MAC and routing layers, both from a general design point of view [1], [2] and by developing specific solutions.

One of the prominent issues in the design of underwater acoustic networks is energy efficiency, since the nodes are often powered by a limited battery supply. In [3] the authors aim at saving energy by introducing a collision avoidance scheme with an RTS/CTS (request-to-send/clear-to-send) handshake. Based on the distance between the transmitter and the receiver, the nodes set a waiting time for DATA transmission in order to reduce the number of collisions. In [4], a MAC algorithm based on sleep cycles is proposed. Every node sends a SYNC packet in order to synchronize its cycle. Transmissions can thus be scheduled in an optimal manner, provided that the network is static so that all the nodes can be synchronized.

Underwater routing problems are addressed in [5], taking into account the relationship between distance, frequency, and transmission power [6]. A geographic routing protocol called the Focused Beam Routing is proposed in [7]. Its purpose is to find shorter hops in order to save energy using different transmission power levels. In [8], solutions based on duty cycle and wake-up radio are compared for the case of fixed transmission power (i.e., no power control).

In this paper we study the effect of node density on the energy consumption in a network where the nodes employ a duty cycle strategy and have the ability to control the transmission power. We assume that each node employs a duty cycle strategy in a random manner, independently of the other nodes. The strategy is a simple one, in which a node is on for a fraction $\alpha$ of a pre-specified cycle length $T_{c}$, and off for the rest of the cycle time. The nodes start their cycles at arbitrary times, so that global synchronization is not necessary. The goal in doing this is to reduce the energy spent in idle listening. However, since the effective number of nodes is reduced (by $\alpha$ on the average), the average inter-node distance increases, requiring an increase in the transmission power. Thus, there is a trade-off between the energy saved on unnecessary listening, and the energy spent in transmission over longer distances. The net effect of applying a duty cycle is to reduce the effective node density. This effective density can be used to set the power control to optimize the energy consumption, similarly as in [7], where the power levels are determined as a function of the (actual) node density. Hence, energy consumption can be optimized for every duty cycle, and the question arises as to whether it is possible to find the optimal duty cycle for which the overall energy minimum will be reached. Focusing on several standard MAC protocols and the routing protocol [7], we conduct a simulation analysis showing that this is indeed possible. The optimal duty cycle is a function of the path loss, the ambient noise level, and the specific hardware power levels used for reception and idle listening. Using these values for a particular system, the duty cycle can easily be computed prior to the system deployment but can also be easily adjusted after deployment. This provides an easy way of controlling the energy consumption in a distributed manner, without any requirements on global synchronization.

The paper is organized as follows. In Section II we summarize the relevant characteristics of acoustic propagation. In Section III we describe the duty cycle and the power control schemes. Results are illustrated in Section IV. Section V summarizes the conclusions.

\section{UnderWATER ACOUSTIC CHANNEL}

The attenuation experienced by an acoustic signal of frequency $f$, traveling over a distance $d$, is given by

$$
A(d, f)=A_{0} d^{k} a(f)^{d}
$$

where $A_{0}$ is a unit-normalizing constant obtained for the reference distance of $1 \mathrm{~m}, k$ is the spreading factor (whose 


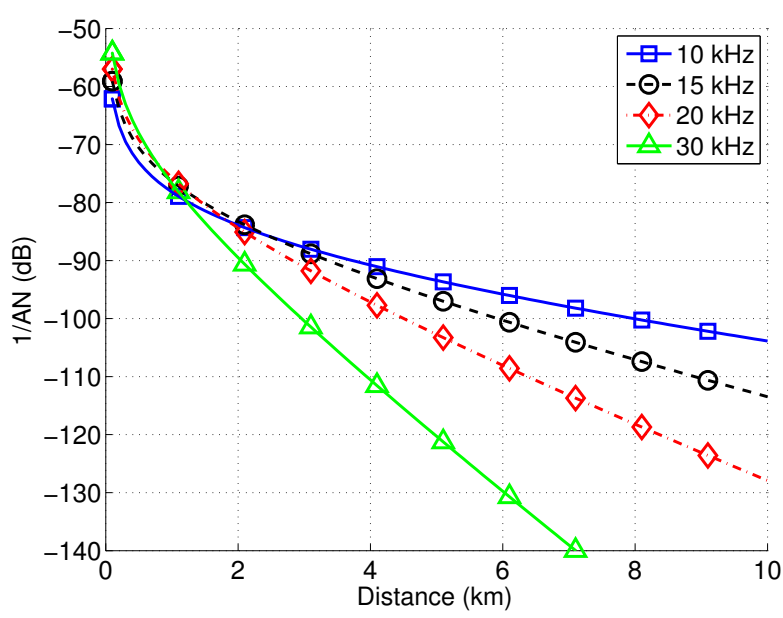

Fig. 1. The factor $1 / A(d, f) N(f)$ as a function of distance for different frequencies.

practical value is taken to be 1.5) and $a(f)$ is the absorption coefficient, given in $\mathrm{dB} / \mathrm{km}$ for $f$ in $\mathrm{kHz}$ by the Thorp formula [6]

$10 \log a(f)=\frac{0.11 f^{2}}{1+f^{2}}+\frac{44 f^{2}}{4100+f^{2}}+2.75 \cdot 10^{-4} f^{2}+0.003$

The absorption coefficient increases rapidly with frequency, limiting the useful acoustic bandwidth. The carrier frequencies used for acoustic communications typically range up to several tens of $\mathrm{kHz}$, with the exact value depending on the transmission distance.

The ambient noise, which is caused by turbulence, distant shipping, surface waves and thermal noise, has a decaying power spectral density that can be approximated as [6]

$$
10 \log N(f) \approx N_{1}-\eta \log f
$$

where $N_{1}$ is a fixed level (we will use $50 \mathrm{~dB}$ re $\mu \mathrm{Pa}$ in the numerical examples) and $\eta=18 \mathrm{~dB} /$ decade.

The combined effect of the frequency-dependent path loss and ambient noise can be described by the behavior of $1 / A(d, f) N(f)$, called the AN factor, which determines the received signal-to-noise ratio (SNR) in a narrow band of frequencies around $f$. The AN factor is shown in Fig. 1.

Another peculiarity of acoustic channels is their long latency. Acoustic waves propagate underwater at $1500 \mathrm{~m} / \mathrm{s}$, much slower than electromagnetic waves in the air.

\section{SYSTEM MODEL}

We consider a bottom-mounted network, covering a square area of side $S$, where $M$ nodes are deployed according to a random grid method as shown in Fig. 2. The area is divided into cells, and a node is placed in each cell randomly, according to a uniform distribution within the cell area. The inter-node distance is thus bounded by $S \sqrt{5 / M}$. The network density is given by

$$
\rho=\frac{M}{S^{2}}
$$

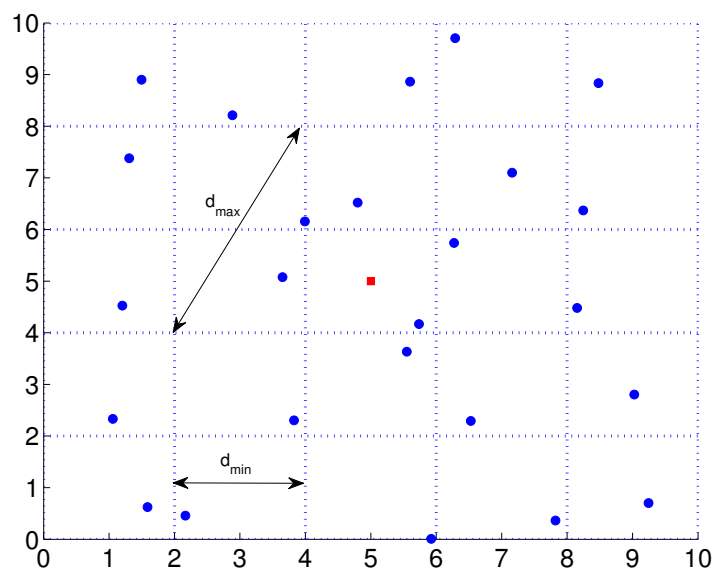

Fig. 2. Network deployment for 25 nodes.

The total network traffic is modeled as a Poisson process with rate $1 / \tau$, where $\tau$ is the mean inter-arrival time between two consecutive packets. Consequently, every node generates packets according to a Poisson process with rate $1 /(M \tau)$, independently of one another. We assume that all the traffic is destined to a common sink, placed in the center of the network.

The nodes are capable of using different power levels, adjusted to span a given set of nominal transmission distances. Routing is implemented using the Focused Beam Routing (FBR) protocol [7], which chooses each new relay towards the sink as the node that can be reached at lowest power.

The duty cycle is defined by $0 \leq \alpha \leq 1$. Given the duration $T_{c}$ of the cycle, every node remains active for $\alpha T_{c}$ seconds, and then switches off the communication interface and becomes unavailable for $(1-\alpha) T_{c}$ seconds. The duration of the cycle is deterministic, and chosen to accommodate a certain number of packets. The nodes apply the duty cycle in an asynchronous manner, each starting at a randomly chosen time. Given the duty cycle $\alpha$, the effective number of nodes is defined as $M_{e f f}=\alpha M$. This is the average number of active nodes in the network, and the corresponding effective node density is

$$
\rho_{e f f}=\frac{M_{e f f}}{S^{2}}=\alpha \cdot \rho .
$$

Every node has a finite number $L$ of power levels ( $L=4$ in our numerical examples). The power levels are set according to the distances

$$
d_{\min }=\frac{S}{\sqrt{M}}
$$

$$
d_{\max }=\min \left(\frac{\sqrt{5}}{\sqrt{\rho_{\text {eff }}}}, S \frac{\sqrt{2}}{2}\right) .
$$

where $d_{\min }$ is the length of the side of a single cell and only depends on the number of nodes in the network, whereas in the $d_{\max }$ formulation, the first term is a generalization of the internode maximum distance in the case of $\alpha<1$ and the second term is the maximum distance from the sink of a generic node, which is equal to half the diagonal of the square area. The range of distances between $d_{\min }$ and $d_{\max }$ is now divided 
into $L$ steps of length

$$
d_{\text {step }}=\frac{d_{\max }-d_{\min }}{L-1}
$$

and the distance corresponding to the $l$-th step,

$$
d_{l}=d_{\text {min }}+l \cdot d_{\text {step }}, l=0, \ldots, L-1
$$

is used to determine the $l$-th power level.

Given the transmission bandwidth $B$ centered around the frequency $f_{c}$, the power needed to span a particular distance $d_{l}$, i.e., to close the link with a pre-specified SNR, $S N R_{0}$, is given by

$$
P_{l}=S N R_{0} \cdot G \cdot \frac{\int_{B} N(f) d f}{\frac{1}{B} \int_{B} A^{-1}\left(d_{l}, f\right) d f}
$$

where we have assumed a flat power spectral density for the transmitted signals. In case of a narrow bandwidth $B$, the above expression simplifies to

$$
P_{l} \approx S N R_{0} \cdot G \cdot A\left(d_{l}, f_{c}\right) N\left(f_{c}\right) \cdot B
$$

The factor $G$ represents the margin that is normally included to account for any additional losses in the system (transducer inefficiencies, increased noise level $N_{1}$, etc.). If the power $P_{l}$ is to be regarded as the electrical power, then the acousticalto-electrical conversion factor $(172 \mathrm{~dB})$ can also be included into the parameter $G$.

In our simulations, we studied many scenarios for different values of $\rho_{e f f}$, obtained varying both the number of nodes, $M$, and the value of the duty cycle parameter, $\alpha$. We want to investigate if applying a duty cycle scheme to the nodes, it is possible to have benefits in terms of energy consumption.

\section{REsults}

To assess the network performance under different duty cycle policies, simulation has been conducted using the AUVNETSim Python simulator [9]. Three simulation scenarios were considered:

1. $S=10 \mathrm{~km}, f_{c}=10 \mathrm{kHz}, B=10 \mathrm{kHz}$ (reference scenario)

2. $S=20 \mathrm{~km}, f_{c}=10 \mathrm{kHz}, B=10 \mathrm{kHz}$ (larger area)

3. $S=10 \mathrm{~km}, f_{c}=30 \mathrm{kHz}, B=10 \mathrm{kHz}$ (higher frequency).

For the other simulation parameters, we set $\tau=60 \mathrm{~s}$, $T_{c}=20 \mathrm{~s}, S N R_{0}=25 \mathrm{~dB}$. Every packet can be retransmitted up to 4 times before being discarded. For the MAC protocol, we have considered Aloha with carrier sensing, carrier sensing multiple access with collision avoidance (CSMA/CA) [10], and the Distance-Aware Collision Avoidance Protocol (DACAP) [3]. Unlike Aloha, CSMA/CA uses an RTS/CTS handshake, and DACAP uses additional waiting times to further decrease the probability of data packet collisions. The energy performance obtained under different MAC protocols was quite similar, so that in what follows we will focus on CSMA/CA only.

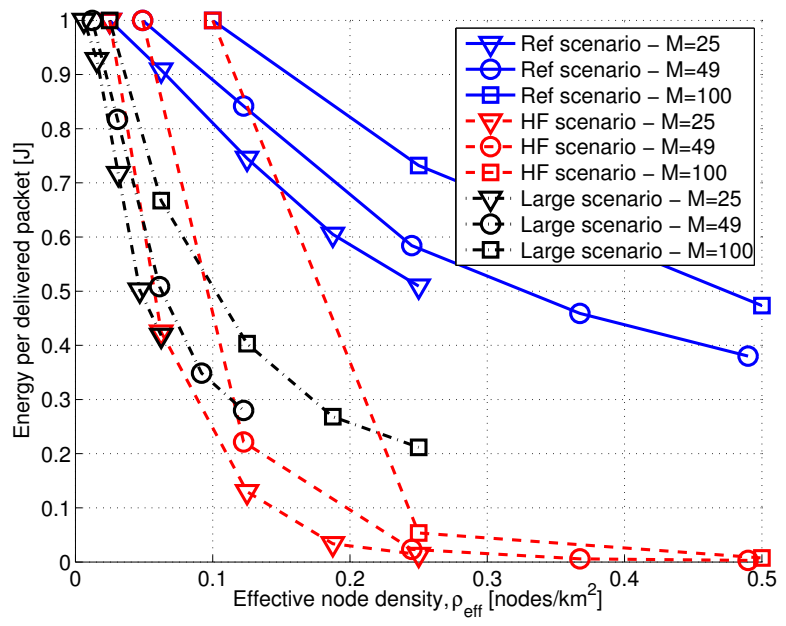

Fig. 3. Normalized transmission energy consumed per delivered packet vs. effective node density, $\rho_{e f f}$, for various scenarios and values of the number of users $M$.

\section{A. Transmission energy}

Fig. 3 shows the energy consumed for transmitting in the different scenarios considered. As expected, the transmission energy decreases as $\rho_{\text {eff }}$ decreases, since the average internode distance becomes shorter.

The effect of increasing the coverage area or the carrier frequency is a faster decrease of the transmission energy with $\rho_{\text {eff }}$. This effect is a consequence of the frequency-anddistance dependence of the acoustic AN factor (Fig. 1). Simply stated, a larger area implies longer inter-node distances for the same density, while higher frequencies experience more absorption. Both situations demand a greater transmission power to close the link with a desired SNR.

\section{B. Reception energy}

Fig. 4 shows the energy consumed for receiving in the different scenarios considered. Unlike transmission, reception power does not depend on the distance between the nodes, but only on the number of nodes in the network. Reception energy thus increases with the effective density $\rho_{e f f}$. Increasing the network area does not affect the reception energy since the number of nodes is kept the same. Since the power levels scale with distance, the number of hops required to reach the sink remains the same, making the receiving energy roughly proportional to $M_{e f f}$.

\section{Total energy}

Total energy consumption depends on the particular power settings used for transmission, reception and idle listening. Assuming that the energy consumed during sleep is negligible, we set the ratio between active reception and idle listening power to $P_{r} / P_{i}=100$. We define the reference reception power as $0.1 \mathrm{~W}$, and the reference transmission power as $1 \mathrm{~W}(172 \mathrm{~dB}$ re $\mu \mathrm{Pa}$ at $1 \mathrm{~m})$. Different transducer efficiencies and link margins, which are embodied in the factor $G$ in the expression (9) will have an effect on the overall energy consumption, as illustrated in Fig. 5. This figure shows the total 


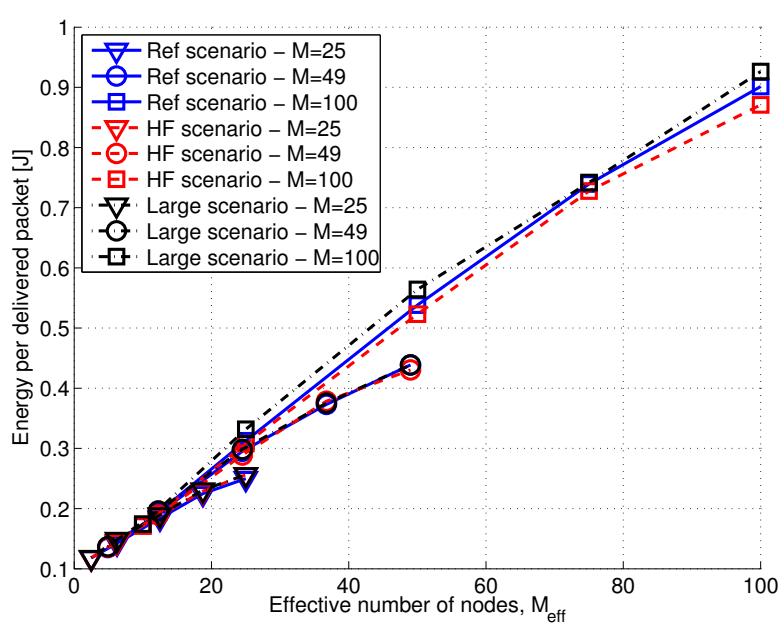

Fig. 4. Reception energy consumed per delivered packet vs. effective number of nodes $M_{e f f}$, for various scenarios and values of the number of users $M$.

energy consumption trend for different values of $G$, ranging from $15 \mathrm{~dB}$ to $23 \mathrm{~dB}$. When $G$ is low, transmission power is used efficiently, and the total energy consumption is dominated by reception, increasing almost linearly with $\rho_{\text {eff }}$. With an increase in fixed losses $G$, the required transmission power increases, and the transmission power begins to dominate. The effect is notably pronounced at low effective densities. As a result, for certain values of $G$, the two energy components are balanced, and it is possible to find an optimum value of $\rho_{\text {eff }}$. In Fig. 5, this occurs for $G=20 \mathrm{~dB}$, where the optimal $\rho_{\text {eff }}$ is 0.25 nodes $/ \mathrm{km}^{2}$ while, as expected, when $G=23 \mathrm{~dB}$ this minimum shifts towards a higher value of $\rho_{e f f}$, around 0.4 nodes $/ \mathrm{km}^{2}$. This value in turn implies the optimal duty cycle to be used in the network.

For a larger coverage area (simulation scenario 2, not shown) an optimal $\rho_{\text {eff }}$ can be found at lower values of $G$, since the inter-node distances are longer and transmission dominates the overall energy consumption. At higher center frequencies (simulation scenario 3, not shown) a similar trend is observed, as greater transmission powers are needed to overcome the absorption.

\section{Network performance}

Besides the energy consumption, other network performance metrics include delay, reliability and collision probability. Below, we discuss these metrics for the different MAC protocols used (Aloha, CSMA/CA, DACAP). While the energy performance depends on $\rho_{e f f}$ almost regardless of the number of nodes $M$ and the duty cycle $\alpha$, the other performance metrics depend on the individual contributions of these two parameters.

1) Delay: Fig. 6 shows the delay performance. Comparing the three curves corresponding to different numbers of nodes, we see that a higher number of nodes implies a longer delay. In general, the delay performance improves with $\alpha$, except when the number of nodes is high and the duty cycle low ( $M=100, \alpha<0.1$ ). In that case, the disadvantages of the low duty cycle are compensated by the network sparseness (the

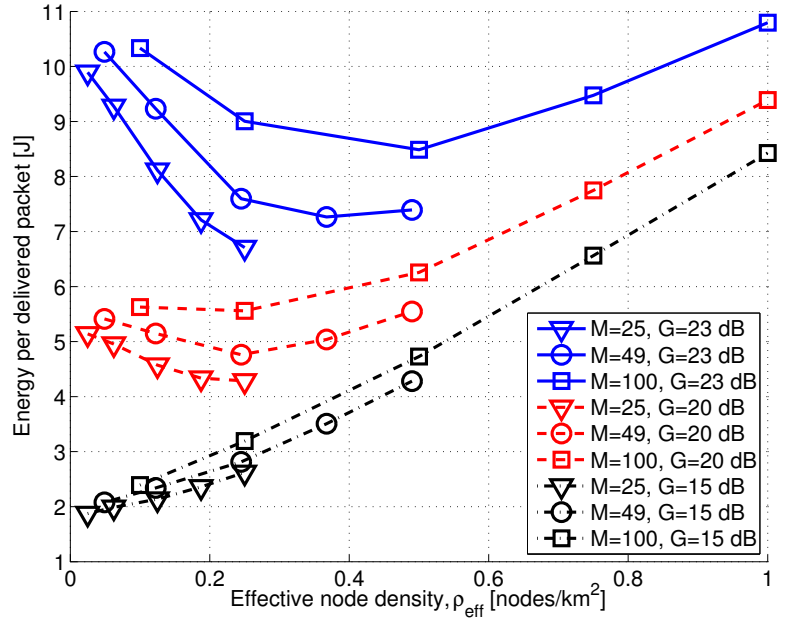

Fig. 5. Total energy consumed per delivered packet vs. effective node density, $\rho_{e f f}$, for the reference scenario and various values of the number of users, $M$, and of the fixed losses, $G$.

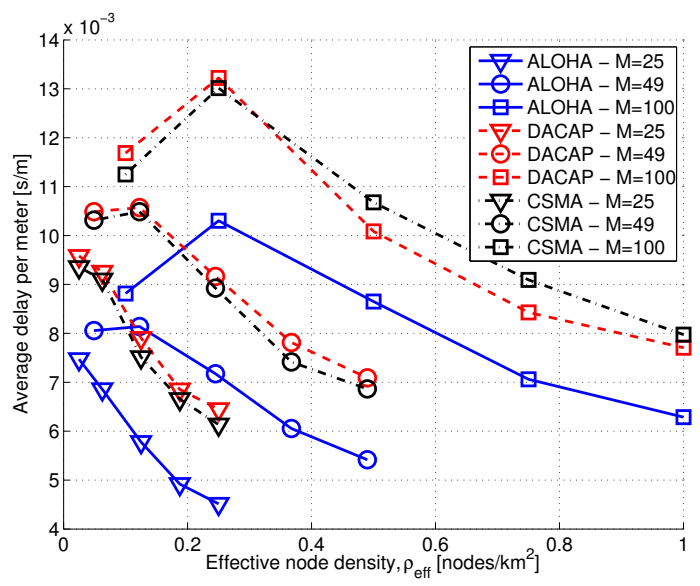

Fig. 6. Average packet delay per meter vs. effective node density, $\rho_{e f f}$, for various MAC protocols and values of the number of users $M$.

average number of hops is close to one, and there are very few collisions). The delay is not influenced by collisions only, but also by the initial waiting (a packet that arrives while the node is asleep has to wait until the node wakes up). A long sleep time (low duty cycle) then implies longer waiting. Therefore, the number of nodes and the duty cycle affect the end-to-end delay independently.

Comparing the performance of different MAC protocols, we observe that Aloha is the fastest. DACAP, with its waiting time to avoid collisions, is the slowest, although its performance is very similar to that of CSMA/CA.

2) Reliability: The reliability of the network is described by the probability that a packet is correctly delivered to the sink. To assess this probability, we measured the packet delivery ratio $\xi$ (the number of packets delivered, divided by the total number of packets generated in the network). In fact, a packet can be retransmitted only a limited number of times (in our simulations we set the maximum retransmissions to 4 ), before it is dropped by the network.

Fig. 7 shows the packet delivery ratio. We see that $\xi$ 


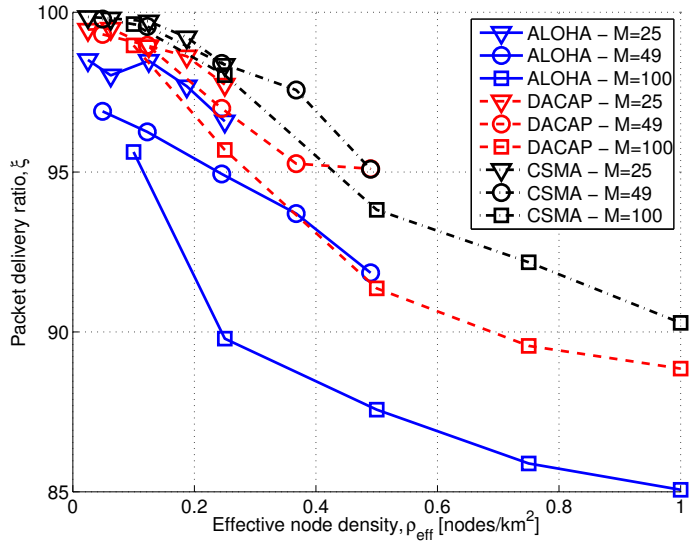

Fig. 7. Packet delivery ratio vs. effective node density, $\rho_{e f f}$, for various MAC protocols and values of the number of users $M$.

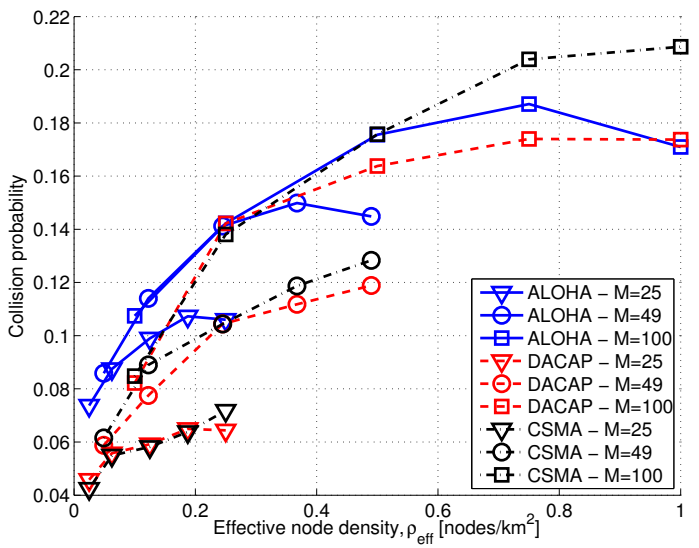

Fig. 8. Data packet collision probability vs. effective node density, $\rho_{e f f}$, for various MAC protocols and values of the number of users $M$.

decreases with $\rho_{e f f}$. This is explained by the increased number of collisions at higher network density.

The reliability is obviously influenced by the choice of the MAC protocol. CSMA/CA now offers the best performance, followed closely by DACAP. These two protocols are quite reliable: even in a very dense network CSMA/CA and DACAP yield $\xi \geq 0.90$ and $\xi \geq 0.89$ respectively, while for low density they result in $\xi \geq 0.96$. Their performance is similar for varying $M$, i.e. it depends mainly on $\rho_{e f f}$. In contrast, Aloha has poor reliability, which also varies with the number of nodes $M$. With a large number of nodes $(M=100)$ and at high duty cycle, only $85 \%$ of the packets are delivered and only for $\alpha=0.1 \xi>0.9$.

3) Collision probability: Packet collisions imply a waste of energy and prolonged delays and, due to the limit on the number of retransmissions, an increase in the number of dropped packets. Collisions are not obvious to an end user, but they influence the energy consumption, delay and reliability, and link these important metrics together.

Fig. 8 shows the measured probability (rate) of packet collisions. As expected, collision probability increases with $\rho_{e f f}$. Aloha exhibits the highest collision rate, except for the highestdensity scenario, while DACAP has the best performance. It is also interesting to note how the duty cycle affects the

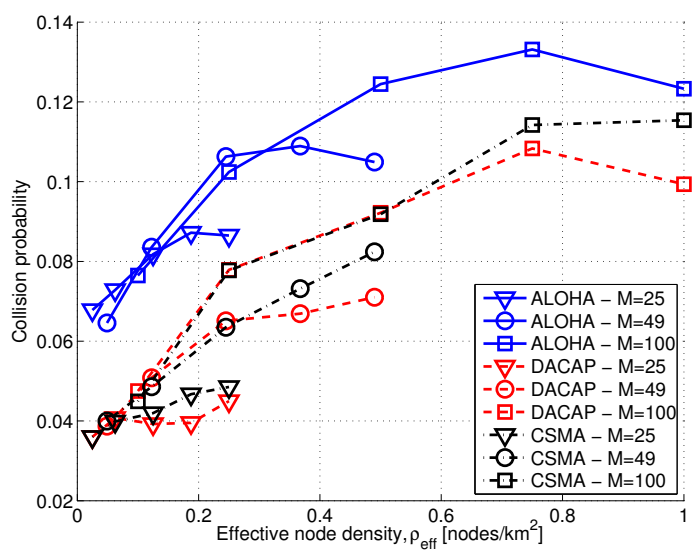

Fig. 9. Data packet collision probability vs. effective node density, $\rho_{e f f}$, in the High frequency scenario, for various MAC protocols and values of the number of users $M$.

performance: given a certain value of $\rho_{e f f}$, fewer nodes and a higher duty cycle result in fewer collisions.

Network performance was also investigated in simulation scenarios with larger coverage area $(S=20 \mathrm{~km})$ and higher frequency $\left(f_{c}=30 \mathrm{kHz}\right)$. For the larger area, the performance in terms of delay, reliability, and collision probability is very similar to the reference scenario. Power control based on distance ensures that the links between the nodes are just scaled, leading to similar network behavior as the coverage area changes. When the center frequency is increased, signals attenuate more rapidly with distance, but the overall effect of absorption is to limit the interference distance. As a result, collision probability is reduced (Fig. 9) and the network performance improves in terms of delay and reliability.

\section{CONCLUSiON}

We studied the possibility of using a duty cycle scheme to control the density of active nodes in an underwater acoustic network. The goal in doing so was to minimize the overall energy consumption in the network. Namely, if the number of active nodes is reduced (because some go to sleep), the power invested in reception will decrease, but the power needed for transmission will increase because of the longer internode distances. If it is possible to strike a balance between these two opposing trends, an overall energy minimum will be reached. We have shown that a simple duty cycle scheme, coupled with power control, can achieve this goal. Simulation results, obtained for a specific choice of network protocols (CSMA/CA and FBR [7]) clearly indicate the existence of an optimal effective node density for which the overall energy consumed per correctly delivered packet is minimized. Since the effective density is the actual network density scaled by the duty cycle factor, $\rho_{e f f}=\alpha \rho$, it is possible to find an optimal duty cycle for a given network. This duty cycle scheme is a very simple one, in which the nodes stay active for a fraction $\alpha$ of a pre-determined, fixed cycle time, and turn off for the remainder of the cycle. The nodes start cycling at arbitrary times, independently of one another, and, hence, global synchronization is not required. 
Network performance under the duty cycle scheme was also assessed in terms of delay, reliability (packet delivery ratio) and collision rate. Lowering the duty cycle was found to yield a longer delay, but an improved reliability and collision rate. The impact of coverage area and center frequency was also studied. Changing the coverage area while keeping the same number of nodes in a network does not affect the performance, since power control ensures a scalable system design. Changing the center frequency, however, was shown to have an impact on the network performance: allocating the system bandwidth to higher frequencies limits the interference range, and boosts the performance in terms of both delay and reliability.

Future work will focus on determining the system performance for varying offered load, determining the relationship between the packet size and the cycle time, and assessing the system performance in time-varying (fading) channels.

\section{ACKNOWLEDGMENTS}

This work was supported in part by the NSF grant 0831728, the ONR grant N00014-09-1-0700, the Italian Institue of Technology under the "Project Seed" program and by MIT Sea Grant College Program, under NOAA grant NA06OAR4170019, project R/RT-2/RCM-21.

\section{REFERENCES}

[1] I. F. Akyildiz, D. Pompili, and T. Melodia, "Underwater acoustic sensor networks: research challenges," Ad Hoc Networks, vol. 3, no. 3, pp. 257 $-279,2005$.

[2] J. Heidemann, W. Ye, J. Wills, A. Syed, and Y. Li, "Research challenges and applications for underwater sensor networking," in IEEE Wireless Communications and Networking Conference, WCNC 2006., vol. 1, April 2006, pp. 228-235.

[3] B. Peleato and M. Stojanovic, "Distance aware collision avoidance protocol for ad-hoc underwater acoustic sensor networks," IEEE Communications Letters, vol. 11, no. 12, pp. 1025 -1027, December 2007.

[4] M. K. Park and V. Rodoplu, "UWAN-MAC: An energy-efficient MAC protocol for underwater acoustic wireless sensor networks," IEEE Journal of Oceanic Engineering, vol. 32, no. 3, pp. 710-720, July 2007.

[5] M. Zorzi, P. Casari, N. Baldo, and A. Harris, "Energy-efficient routing schemes for underwater acoustic networks," IEEE Journal on Selected Areas in Communications, vol. 26, no. 9, pp. 1754-1766, December 2008.

[6] M. Stojanovic, "On the relationship between capacity and distance in an underwater acoustic communication channel," ACM SIGMOBILE Mob. Comput. Commun. Rev., vol. 11, no. 4, pp. 34-43, 2007.

[7] J. M. Jornet, M. Stojanovic, and M. Zorzi, "Focused beam routing protocol for underwater acoustic networks," in WuWNeT '08: Proceedings of the third ACM international workshop on Underwater Networks. New York, NY, USA: ACM, 2008, pp. 75-82.

[8] A. F. Harris, III, M. Stojanovic, and M. Zorzi, "When underwater acoustic nodes should sleep with one eye open: idle-time power management in underwater sensor networks," in WUWNet '06: Proceedings of the 1st ACM international workshop on Underwater networks. New York, NY, USA: ACM, 2006, pp. 105-108.

[9] J. Montana, "AUVNetsim: A simulator for underwater acoustic networks," MIT Sea Grant Technical Report, 2008. [Online]. Available: http://users.ece.gatech.edu/jmjm3/publications/auvnetsim.pdf

[10] D. Bertsekas and R. Gallager, Data networks. Prentice Hall, 1992. 